\title{
Plasma-based processes for planar and 3D surface patterning of functional nanoparticles
}

\author{
S. Askari $\mathbb{D} \cdot$ H. Machhadani • J. Benedikt • \\ U. Helmersson
}

Received: 19 August 2019/Accepted: 25 September 2019/Published online: 30 October 2019

(C) The Author(s) 2019

\begin{abstract}
We present a gas-phase process for surface patterning and 3D self-assembly of nanoparticles (NPs) of functional materials such as metals, oxides, and nitrides. The method relies on electrostatic assembly of free-flying NPs with unipolar charge produced in plasma sources. We demonstrate the capability of the process in self-assembly of NPs, with the size in the range 10-60 nm, into arrays of free-standing 3D microstructures with complex morphologies. Considering that the plasma nanoparticle sources are compatible with synthesis of a large library of material NPs, the process introduces a novel approach for 3D printing of various functional NPs, high-precision device integration of NPs on sub-micrometer scales, and large-area parallel surface patterning of NPs.
\end{abstract}

Keywords Plasma $\cdot$ Sputtering $\cdot$ Functional nanoparticles $\cdot 3 \mathrm{D}$ assembly

Electronic supplementary material The online version of this article (https://doi.org/10.1007/s11051-019-4674-3) contains supplementary material, which is available to authorized users.

S. Askari · H. Machhadani · U. Helmersson

Plasma \& Coatings Physics Division, IFM-Material Physics,

Linköping University, SE-581 83 Linköping, Sweden

S. Askari $(\bowtie) \cdot J$. Benedikt

Institute for Experimental and Applied Physics,

Christian-Albrechts-Universität zu Kiel, Leibnizstraße 17,

24118 Kiel, Germany

e-mail: askari@physik.uni-kiel.de

\section{Introduction}

Directed self-assembly of functional nanoparticles (NPs) has emerged as a powerful bottom-up nano-synthesis approach for creating novel functional materials and surfaces, integrating NPs in miniaturized devices, and also 3D printing of functional materials (Boles et al. 2016; Grzelczak et al. 2010; Yang et al. 2016; Flauraud et al. 2017; Gwo et al. 2016; Huang et al. 2016; Su et al. 2014). A variety of devices have been reported using NP assembly techniques including novel optoelectronic devices (e.g., light-emitting diodes, sensors, solar cells, photodetectors, and transistors), optical and plasmon sensing devices, electrodes in nanoscale electrochemistry, printable chips, nano-pore biosensors, and transparent electrodes (Grzelczak et al. 2010; Yang et al. 2016; Flauraud et al. 2017; Gwo et al. 2016; Huang et al. 2016; Su et al. 2014; Lewis and Ahn 2015; Choi et al. 2016; Xie et al. 2015; Ellis et al. 2014; Momotenko et al. 2016; Blasco et al. 2016). In response to the growing demand in the field, diverse techniques have been developed for 3D self-assembly of ligand-protected colloidal NPs including lithographic patterning, template-based methods, DNA-mediated assembly, dip-pen processes, and capillary assembly (Su et al. 2014; Xie et al. 2015), and also for 2D surface patterning such as etching of close-packed structures and atomic layer deposition (ALD) (Wang et al. 2018; Dendooven et al. 2017; Schmudde et al. 2016; Hou et al. 2018). In 3D printing technology, NPs are now widely used for 3D printing in ink-jet printers that are compatible with printing polymer and metals using colloidal inks (Hirt et al. 2017). 
The general issues limiting the techniques are the complexity of the NP synthesis (by multi-step chemical methods) and its coupling into the assembly technique, the complexity of the assembly techniques, limited control over geometry especially in 3D architectures, limitations involved with the range of materials, and also limited spatial resolution of the methods.

In a different approach, free-flying NPs produced in a flow-through gas-phase process can be directed onto desired positions with a high spatial resolution (Ma et al. 2007; Fonzo et al. 2000; Popok et al. 2011; Vece 2019; Wegner et al. 2012; Barborini et al. 2008). A common approach is to focus the beam of NPs, produced in the gas phase, through an aerodynamic lens apparatus that allows localized deposition of NPs. Among the gas-phase methods, non-thermal plasma processes have emerged as a viable approach for synthesis of NPs of various functional materials (Kortshagen et al. 2016). NPs extracted from plasmas are negatively charged, which leads to the suppression of NP agglomeration, a serious issue in other gas-phase production methods. Furthermore, it is feasible to use long-range forces such as electric and/or magnetic forces (for magnetic nanoparticles) to manipulate NP trajectory toward the substrate (Ekeroth et al. 2018).

The idea of flow-through high-resolution 3D selfassembly of functional NPs pursued here relies on efficient charging of free-flying NPs and their directed positioning using a designed electric-field configuration placed on a substrate in the vicinity of the assembly zone. In the present work, we employ a hollow cathode pulsed plasma sputtering technique for controlled growth of NPs. In the plasma, the high mobility of the electrons will cause the NPs to become negatively charged. Highly reactive plasma chemistry is generated in the plasma because of the large population of energetic electrons while the gas temperature remains close to ambient temperature. Fast growth of NPs occurs because of the low temperature of the plasma and rapid accumulation of ions onto negatively charged NPs. The simplicity of sputtering from a solid target provides access to a vast library of functional materials including metals, alloys, oxides, and nitrides. Our previous studies show that the method is highly flexible in terms of control over size, chemical composition, and optoelectronic properties of the NPs (Ekeroth et al. 2018; Askari et al. 2018; Pilch et al. 2013; Gunnarsson et al. 2015; Ekeroth et al. 2019).

\section{Experimental process}

The plasma process used in the present experiments is shown schematically in Fig. 1a. The cathode consists of a cylindrical tube (inside diameter $5 \mathrm{~mm}$ and length 55 $\mathrm{mm}$ ) made from the solid precursor material (e.g., Ag). The cylinder is installed in a water-cooled holder with electric and gas fittings (see SI document and our previous report (Askari et al. 2018) for details of the experimental setup). The carrier gas (e.g., Ar) flows through the hollow cathode with a flow rate in the range of 60 $80 \mathrm{sccm}$. In some experiments, an additional reactive gas (e.g., $\mathrm{N}_{2}, \mathrm{O}_{2}$ ) is supplied, either mixed in with the carrier gas or supplied separately in a vacuum system. A grounded anode ring is placed $30 \mathrm{~mm}$ below the cathode aperture. The plasma is formed by applying a pulsed electric voltage to the cathode. Ions generated in the discharge are accelerated toward the cathode surface and erode (or sputter) the cathode and eject the material out into the plasma region outside the hollow cathode. The electric energy is applied in the form of short intense pulses with a pulse duration of $80 \mu$ s and a repetition frequency of $1200 \mathrm{~Hz}$ which lead to a high degree of ionization of the sputtered atoms that are puffed out of the cathode into the plasma volume outside the hollow cathode.

In non-thermal low-pressure plasmas, electrons have significantly higher thermal velocity than ions because of their higher temperature and lower mass. The electron temperature $\left(T_{\mathrm{e}}\right)$ in non-thermal plasmas is typically higher than $1 \mathrm{eV}$ (e.g., $1-5 \mathrm{eV}$ ), while the ion temperature could be as low as room temperature (typically lower than 0.1 $\mathrm{eV}$ ). For an object floating inside a plasma, the net (ion + electron) current to the object is 0 . Therefore, the electric potential of a floating object relative to the plasma will remain negative so that the excess collection of electrons is retarded. A typical potential for a flat surface (i.e., a wall) is $\sim 4.7 T_{\mathrm{e}} \mathrm{eV}$ and for a spherical nanoparticle around $2.4 T_{\mathrm{e}}$ $\mathrm{eV}$ (Kortshagen et al. 2016). Operating in a vacuum brings the advantage of efficient charging of NPs in plasma and preserving the charge over long distance from the plasma. The nucleation and growth of InN NPs inside the plasma have been discussed in our previous report (Askari et al. 2018). We expect that a large percentage of the sputtered material are converted into NPs depending on the operating parameters. Owing to the NP charging, positive ions are collected into the growing NPs significantly faster than the neutral atoms. Apart from the NPs, any surface in contact with the plasma will acquire electric charges from 

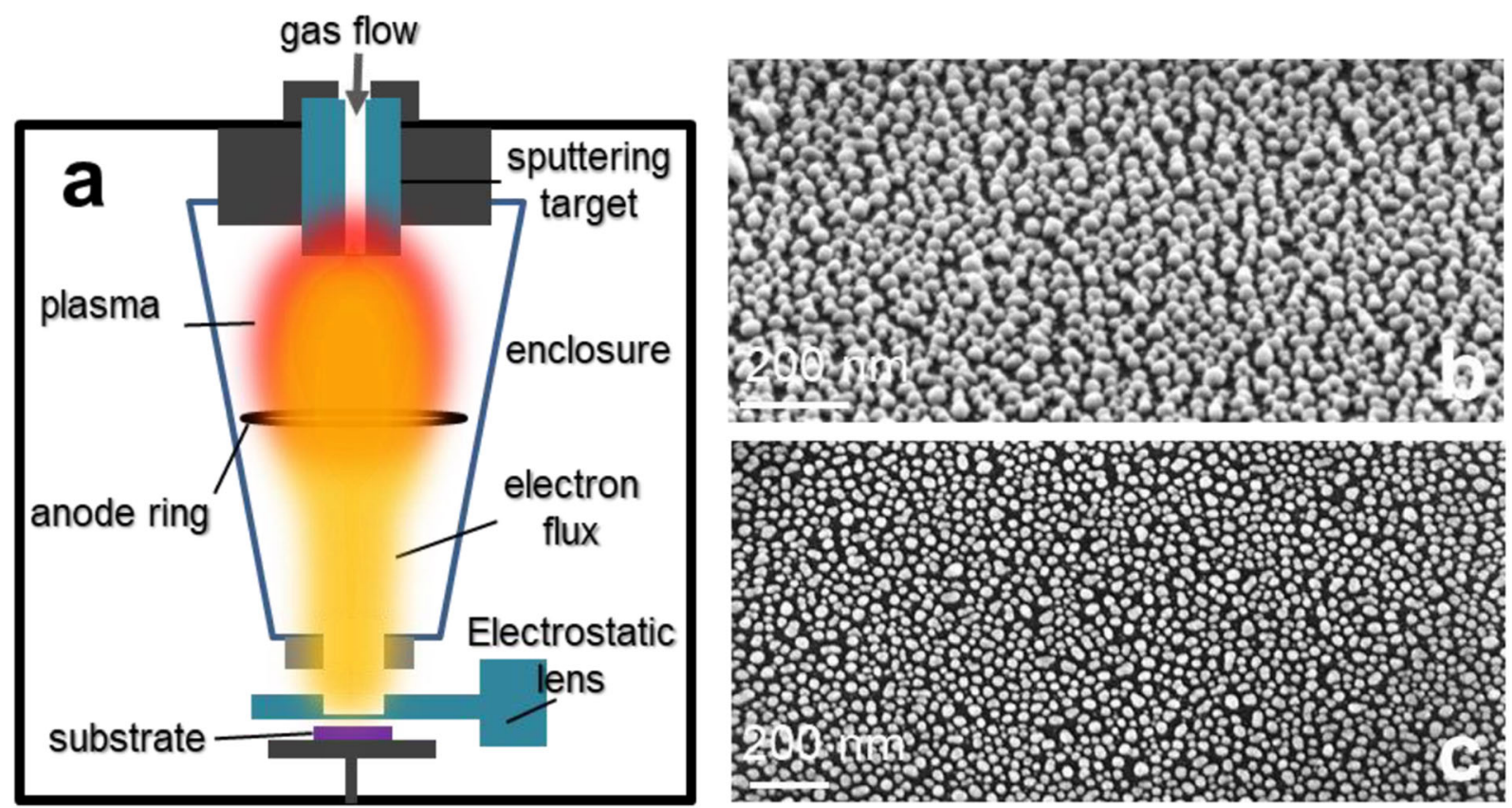

Fig. 1 a Schematic of the experimental setup used for growth and self-assembly of nanoparticles; $\mathbf{b}$ and $\mathbf{c}$ SEM images of Ag NPs formed on the Si substrate

it, which can be beneficially used for self-assembly of NPs onto the substrate.

An electrically isolated enclosure around the plasma was used for guiding NPs toward the substrate (Fig. 1a). The enclosure is charged by plasma and therefore helps guide NPs toward the substrate. Several techniques are used for characterizing synthesized NPs of $\mathrm{Ag}, \mathrm{InN}$, and $\mathrm{ZnO}$. It includes scanning electron microscopy (SEM) and X-ray diffraction (XRD) for material characterization, photoluminescence (PL) and UV-visible absorption spectroscopy for optical characterization, and also electrical measurements of the NPs (see SI document).

\section{Results and discussion}

Figure $1 \mathrm{~b}$ and $\mathrm{c}$ show SEM images of Ag NPs deposited on electrically isolated silicon wafers. For preparing the samples, silicon substrate is placed $5 \mathrm{~cm}$ below the anode ring inside the metal enclosure. The deposition time was 30 s. Deposition of NPs formed in the plasma on the substrate is not expected as the substrate is negatively charged. It is likely that ultra-small $\sim 1$-nm nanoparticles and clusters formed inside the plasma that are not necessarily negatively charged play a major role in formation of such isolated nanoparticles on the substrate.
Surfaces covered with non-close-packed 2D arrangement of NPs are attractive for applications such as optical devices, bio-medical sensors, and catalysis. Several methods have been reported for preparation of such functional surfaces including colloidal lithography and atomic layer deposition (ALD) (Dendooven et al. 2017; Schmudde et al. 2016; Hou et al. 2018). Interestingly, our plasma sputtering method is capable of rapid and largearea deposition of such non-close-pack arrays of NPs.

Charged NPs extracted from the enclosure (Fig. 1a) are positioned on the substrate precisely by applying a predesigned configuration of the electric field. Two different approaches are examined and will be discussed here: (i) NPs are positioned on an electrically charge-patterned substrate, and (ii) guiding NPs through an external electric field assembly above the substrate surface.

Figure 2a illustrates the process for localized positioning of NPs on a substrate pre-patterned by a layer of photoresist (PR). Sample preparation followed three steps: (i) photoresist is spin coated on the substrate using lithography techniques; (ii) NPs are collected on the substrate; (iii) PR is washed away using a solvent. The SEM image shown in Fig. $2 b$ is for the sample prepared by the substrate bias voltage of $17.0 \mathrm{~V}$. The current of electrons from the plasma electrostatically charges the area covered with the insulating polymer on the substrate. As a result, the flux of negatively charged NPs approaching the surface is 


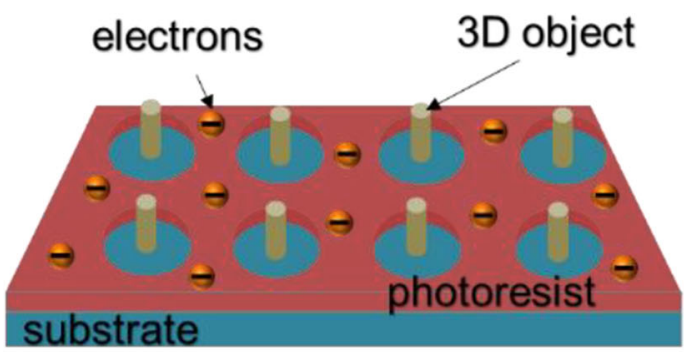

InN nanoparticles

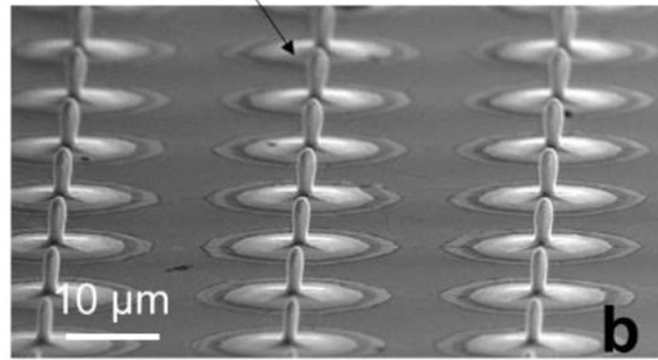

Fig. 2 a Schematic of the growth of 3D pillars inside the openings on a pre-patterned substrate. Electrons from plasma charge the surface of the photoresist. b SEM image shows the 3D pillars of

funneled into the charge-dissipating opening on the substrate. Lithography is a powerful technique in 2D surface pattering of substrate so that any desired shape for the opening is achievable in large arrays. Arrays of circles were pre-patterned on the surface that lead to the growth of pillars perpendicular to the substrate surface (Fig. 2b). The width of the pillar is $\sim 10$ times smaller than the opening diameter due to the focusing of the NPs, i.e., the trace of the removed PR can still be seen in the SEM image in Fig. 2b. The height of the pillars is roughly the same as the PR thickness. Therefore, the pillar width remains constant during the growth as the growth occurs fully inside the opening hole.

In a similar approach, silver NPs with the size of around $30 \mathrm{~nm}$ are positioned on the apex of InGaN pyramids (Fig. 2c, d). The arrays of pyramids that have been fabricated by Houssaine Machhadani et al. were utilized as localized single-photon emitters (Jemsson et al. 2014). The efficiency of the device is expected to enhance with the plasmonic Ag NPs positioned on the tip of the pyramids. The preparation process of the sample is summarized in three steps: (i) the area between the pyramids is spin coated by photoresist with the pyramid tips staying uncovered; (ii) NPs are collected
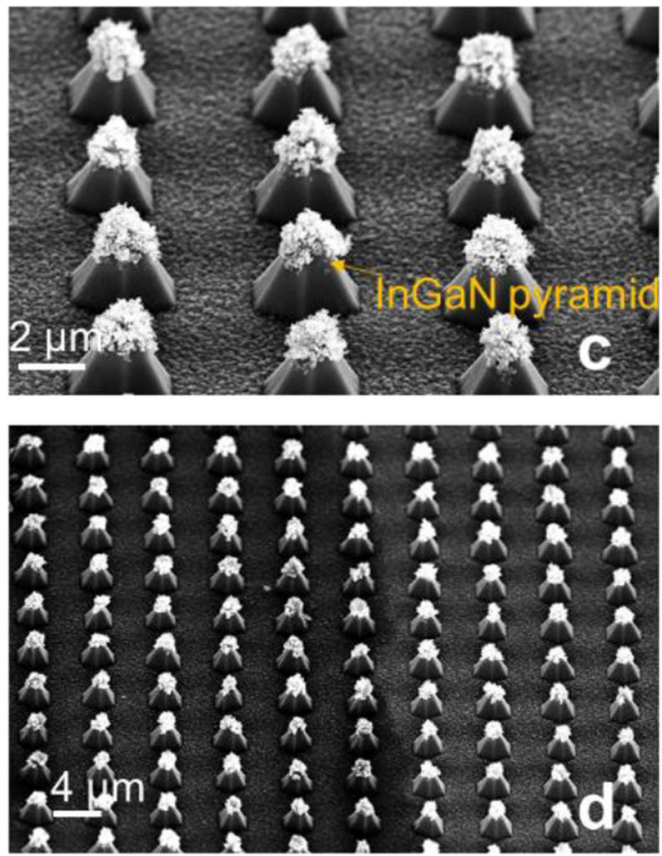

InN nanoparticles. c and $\mathbf{d ~ A g ~ n a n o p a r t i c l e s ~ p o s i t i o n e d ~ o n ~ t h e ~}$ apex of InGaN pyramids using electrostatic self-assembly process.

during $45 \mathrm{~s}$ on the substrate biased to $+22 \mathrm{~V}$; (iii) photoresist is washed away using solvent.

The dependency of the patterning process on lithography restrains the method applicability due to the inherent limitations associated with the lithography, i.e., in addition to the cost and cumbersome preparation efforts, lithography is a $2 \mathrm{D}$ fabrication technique and hence it provides limited control over morphology in growth of 3D objects. In a different approach, NPs are guided to the desired spot on the substrate using a designed configuration of the electric field above the surface. A practically simple case is to use a stencil with arrays of holes placed directly on the substrate. To examine this idea, we used a stencil fabricated from electrically insulating materials, and it is simply placed on the substrate i.e., the stencil is negatively charged while it is exposed to the plasma. Figure 3a shows an example of a large array of pillars patterned by using a stencil fabricated from a thin sheet of silicon oxide with arrays of holes prepared by lithography and etching methods (Fig. S6). Figure 3a shows that the NPs focused through the apertures are collected on the substrate in the form of vertical growing pillars. It is notable that the width of the pillars $(\sim 2 \mu \mathrm{m})$ is 20 times smaller than the aperture diameter because of the focusing effect of the charged 


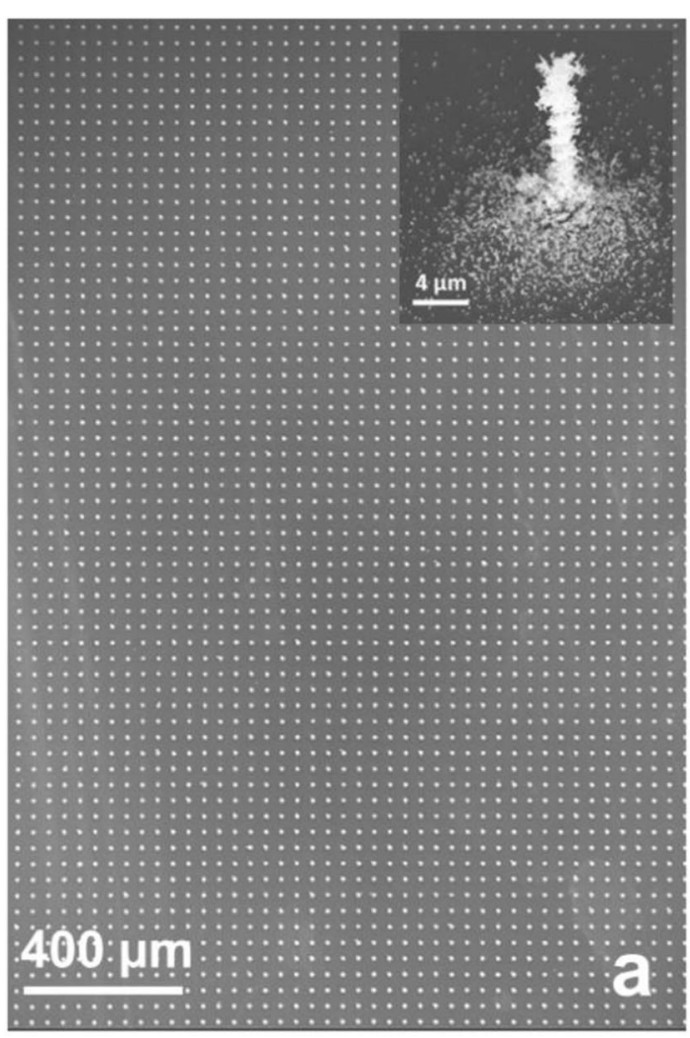

Fig. 3 a and $\mathbf{b}$ SEM image of large-area arrays of 3D pillars of Ag NPs deposited using a stencil placed on the substrate. The inset in a shows a single pillar, where the width of the pillar is around 20

stencil. Figure $3 b$ shows the SEM image of the pillars prepared similarly by a stencil while the substrate is placed $22 \mathrm{~mm}$ below the enclosure aperture.

Further control over the morphology of the 3D features is feasible by using an electrically conductive stencil detached from the surface. The width of the growing pillar is adjustable by changing the voltage applied to the stencil. Figure $3 \mathrm{c}$ and $\mathrm{d}$ show examples whereas the width of the growing column is controlled by changing the applied voltage to the stencil during the deposition process. The width of the features increases so that the overhanging structures with high aspect ratios are formed. In principle, self-assembly of NPs into any axisymmetric 3D morphology is achievable by varying the electric field. 3D features with ultra-high aspect ratios are achievable by adjusting the gap between the substrate and the stencil and/or the stencil thickness. Features with even more complex morphologies are achievable by moving the substrate during the deposition in all three dimensions.
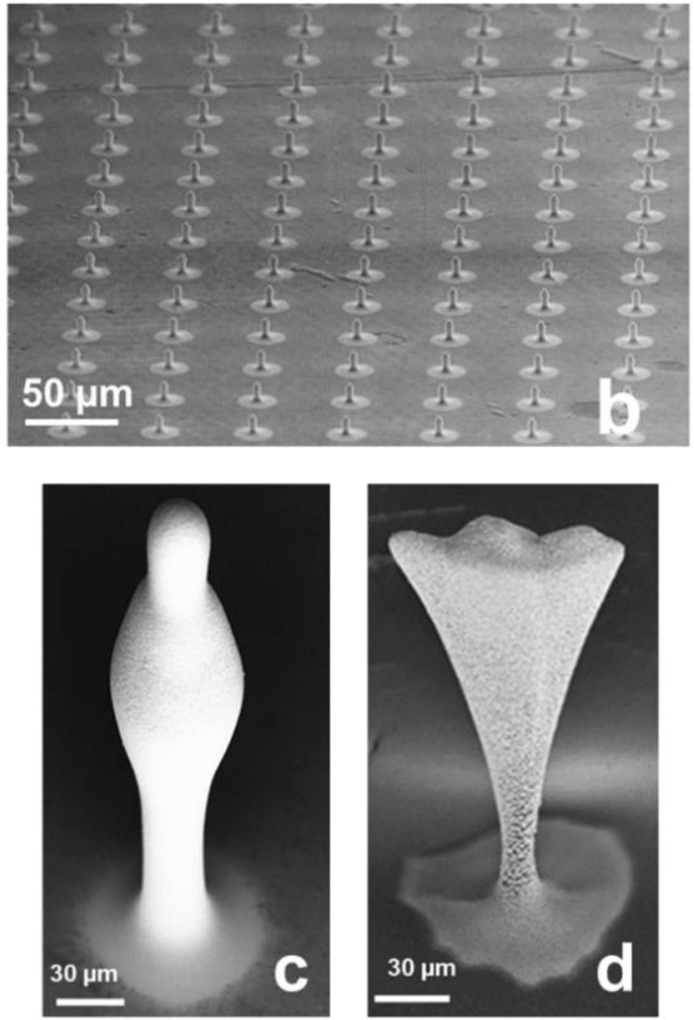

times smaller than the stencil opening; $\mathbf{c}$ and $\mathbf{d}$ pillars of ZnO NPs prepared using electrically biased templates with two different bias voltages of $-0.5 \mathrm{~V}$ and $-1.2 \mathrm{~V}$, respectively.

\section{Conclusion}

In summary, NPs of functional materials produced in a plasma source could be assembled into planar patterns or complex 3D structures. Large arrays of 3D features with high aspect ratios are built simply by using a stencil placed on the substrate and exposed to the electron flux from the plasma. Using the combination of long-range electric forces and $3 \mathrm{D}$ piezo motion, the process will allow printing features with complex morphologies that are not easily achievable with conventional techniques. Plasma nanoparticle sources equipped with such electrostatic lenses would be capable of rapid and highresolution surface patterning of NPs with possible application in device fabrication and $3 \mathrm{D}$ printing of functional materials in sub-microscales.

Acknowledgments We would like to thank Dr. K. Fredrik Karlsson for his help on preparation of substrates with arrays of GaN micropyramids at Linköping University. 
Funding information Open access funding provided by Linköping University. The authors received financial support from the Knut and Alice Wallenberg Foundation (grant KAW 14.0276) and the Swedish Government Strategic Research Area in Materials Science on Functional Materials at Linköping University (faculty grant SFO-Mat-LiU no. 2009-00971). SA received financial support from ÅForsk Foundation (Swedish grant no. 17-517).

\section{Compliance with ethical standards}

Conflict of interest The authors declare that they have no conflict of interest.

Open Access This article is distributed under the terms of the Creative Commons Attribution 4.0 International License (http:// creativecommons.org/licenses/by/4.0/), which permits unrestricted use, distribution, and reproduction in any medium, provided you give appropriate credit to the original author(s) and the source, provide a link to the Creative Commons license, and indicate if changes were made.

\section{References}

Askari S, Mariotti D, Stehr JE, Benedikt J, Keraudy J, Helmersson U (2018) Low-loss and tunable localized mid-infrared plasmons in nanocrystals of highly degenerate InN. Nano Lett 18:5681-5687

Barborini E, Vinati S, Leccardi M, Repetto P, Bertolini G, Rorato O, Lorenzelli L, Decarli M, Guarnieri V, Ducati C, Milani M (2008) Batch fabrication of metal oxide sensors on microhotplates. J Micromech Microeng 18:055015-055022

Blasco E, Müller J, Müller P, Trouillet V, Schön M, Scherer T, Kowollik CB, Wegener M (2016) Fabrication of conductive $3 \mathrm{D}$ gold-containing microstructures via direct laser writing. Adv Mater 28:3592-3595

Boles MA, Engel M, Talapin DV (2016) Self-assembly of colloidal nanocrystals: from intricate structures to functional materials. Chem Rev 116:11220-11289

Choi JH, Wang H, Oh SJ, Paik T, Sung P, Sung J, Ye X, Zhao T, Diroll BT, Murray CB, Kagan CR (2016) Exploiting the colloidal nanocrystal library to construct electronic devices. Science 352:205-208

Dendooven J, Ramachandran RK, Solano E, Kurttepeli M, Geerts L, Heremans G, Rongé J, Minjauw MM, Dobbelaere T, Devloo-Casier K, Martens JA, Vantomme A, Bals S, Portale G, Coati A, Detavernier C (2017) Independent tuning of size and coverage of supported Pt nanoparticles using atomic layer deposition. Nat Commun 8:1074-1086

Ekeroth S, Münger EP, Boyd R, Ekspong J, Wågberg T, Edman L, Brenning N, Helmersson U (2018) Catalytic nanotruss structures realized by magnetic self-assembly in pulsed plasma. Nano Lett 18:3132-3137

Ekeroth S, Ikeda S, Boyd RD, Shimizu T, Helmersson U (2019) Growth of semi-coherent $\mathrm{Ni}$ and $\mathrm{NiO}$ dual-phase nanoparticles using hollow cathode sputtering. J. Nano Res 21:37-45
Ellis BL, Knauth P, Djenizian T (2014) Three-dimensional selfsupported metal oxides for advanced energy storage. Adv Mater 26:3368-3397

Flauraud V, Mastrangeli M, Bernasconi GD, Butet J, Alexander DTL, Shahrabi E, Martin OJF, Brugger J (2017) Nanoscale topographical control of capillary assembly of nanoparticles. Nat Nanotechnol 12:73-80

Fonzo FD, Gidwani A, Fan MH, Neumann D, Iordanoglou DI, Heberlein JVR, McMurry PH, Girshick SL (2000) Focused nanoparticle-beam deposition of patterned microstructures. Appl Phys Lett 77:910-912

Grzelczak M, Vermant J, Furst EM, Liz-Marzan LM (2010) Directed self-assembly of nanoparticles. ACS Nano 4: 3591-3605

Gunnarsson R, Helmersson U, Pilch I (2015) Synthesis of titanium-oxide nanoparticles with size and stoichiometry control. J Nanopart Res 17:353-364

Gwo S, Chen HY, Lin MH, Sunc L, Li X (2016) Nanomanipulation and controlled self-assembly of metal nanoparticles and nanocrystals for plasmonics. Chem Soc Rev 45:5672-5716

Hirt L, Reiser A, Spolenak R, Zambelli T (2017) Additive manufacturing of metal structures at the micrometer scale. Adv Mater 29:1604211-1604241

Hou Y, Wang Z, Cai C, Hao X, Li D, Zhao N, Zhao Y, Chen L, Ma $\mathrm{H}, \mathrm{Xu} \mathrm{J}$ (2018) Conformal nanocoatings with uniform and controllable thickness on microstructured surfaces: a general assembly route. Adv Mater 30:1704131-1704137

Huang Y, Li W, Qin M, Zhou H, Zhang X, Li F, Song Y (2016) Printable functional chips on nanoparticle assembly. Small 13:1503339-1503356

Jemsson T, Machhadani H, Karlsson KF, Hsu CW, Holtz PO (2014) Linearly polarized single photon antibunching from a site-controlled InGaN quantum dot. Appl Phys Lett 105: 081901-081905

Kortshagen UR, Sankaran RM, Pereira RN, Girshick SL, Wu JJ, Aydil ES (2016) Nonthermal plasma synthesis of nanocrystals: fundamental principles, materials, and applications. Chem Rev 116:11061-11127

Lewis JA, Ahn BY (2015) Three-dimensional printed electronics. Nature 518:42-43

Ma LC, Subramanian R, Huang HW, Ray V, Kim CU, Koh SJ (2007) Electrostatic funneling for precise nanoparticle placement: a route to wafer-scale integration. Nano Lett 7:439445

Momotenko D, Page A, Adobes-Vidal M, Unwin PR (2016) Write-read 3D patterning with a dual-channel nanopipette. ACS Nano 10:8871-8878

Pilch I, Söderström D, Brenning N, Helmersson U (2013) Sizecontrolled growth of nanoparticles in a highly ionized pulsed plasma. Appl Phys Lett 102:033108-033112

Popok VN, Barke I, Campbell EB, Meiwes-Broer KH (2011) Cluster-surface interaction: from soft landing to implantation. Surf Sci Rep 66:347-377

Schmudde M, Grunewald C, Goroncy C, Noufele CN, Stein B, Risse T, Graf C (2016) Controlling the interaction and nonclose-packed arrangement of nanoparticles on large areas. ACS Nano 10:3525

Su B, Zhang C, Chen S, Zhang X, Chen L, Wu Y, Nie Y, Kan X, Song Y, Jiang L (2014) A general strategy for assembling nanoparticles in one dimension. Adv Mater 26:2501-2507 
Vece D (2019) Using nanoparticles as a bottom-up approach to increase solar cell efficiency. KONA Powder Part J 36:72-87

Wang K, Ling H, BaoY YM, Yang Y, Hussain M, Wang H, Zhang L, Xie L, Yi M, Huang W, Xie X, Zhu J (2018) A centimeterscale inorganic nanoparticle superlattice monolayer with non-close-packing and its high performance in memory devices. Adv Mater 30:1800595-1800603

Wegner K, Vinati S, Piseri P, Antonini A, Zelioli A, Barborini E, Ducati C, Milani P (2012) High-rate production of functional nanostructured films and devices by coupling flame spray pyrolysis with supersonic expansion. Nanotechnology 23: 185603-185615
Xie W, Gomes R, Aubert T, Bisschop S, Zhu Y, Hens Z, Brainis E, Thourhout DV (2015) Nanoscale and single-dot patterning of colloidal quantum dots. Nano Lett 15:7481-7487

Yang J, Choi MK, Kim DH, Hyeon T (2016) Designed assembly and integration of colloidal nanocrystals for device applications. Adv Mater 28:1176-1207

Publisher's note Springer Nature remains neutral with regard to jurisdictional claims in published maps and institutional affiliations. 